\title{
SOME CHARACTERIZATIONS OF THE FREUDENTHAL COMPACTIFICATION OF A SEMICOMPACT SPACE
}

\author{
R. F. DICKMAN, JR.
}

Throughout this paper let $X$ denote a semicompact Hausdorff space (i.e. every point of $X$ has arbitrarily small neighborhoods with compact boundaries). For completeness we include the definition of the Freudenthal compactification of $X$ [3] as formulated by $\mathrm{K}$. Morita in [4]. Let $\mathfrak{T}$ be the set of all finite open coverings $\left\{G_{1}, G_{2}, \cdots, G_{n}\right\}$ of $X$ such that $F_{r} G_{i}$ are compact. Then $X$ is a completely regular space and $\mathfrak{T}$ is a completely regular uniformity of $X$ agreeing with the topology of $X$. Let $S$ be the completion of $X$ with respect to $\mathfrak{T}$. Then $S$ is a compact Hausdorff space containing $X$ as a dense subset having the following properties:

(a) For any point $x$ of $S$ and any open set $R$ of $S$ containing $x$ there is an open set $V$ of $S$ containing $x$ such that $V \subset R$ and $F r V \subset X$.

(b) Any two disjoint closed subsets of $X$ with compact boundaries have disjoint closures in $S$.

$\mathrm{K}$. Morita has shown that $S$ is the topologically unique Hausdorff compactification of $X$ satisfying (a) and (b), (i.e. if $C$ is any compact Hausdorff space containing $X$ as a dense subset and if $C$ satisfies (a) and (b) then there is a homeomorphism of $S$ onto $C$ that leaves points of $X$ fixed) [4]. This topologically unique space $S$ is denoted by $\gamma X$ and is called the Freudenthal compactification of $X$ after H. Freudenthal who first defined it [3]. For further properties see [3], [4] or [6].

The purpose of this paper is to characterize $\gamma X$ by means of certain minimum and maximum properties similar to those which characterize the Stone-Čech compactification of $\beta X$ of $X$.

First we need some definitions. Let $a$ denote the set of all mappings of $X$ in to $I=[0,1]$. For each $f \in Q$, let $B(f)=\left\{t \in I: F r f^{-1}(t)\right.$ contains a compact set $K$ that separates $X$ into two disjoint open sets $M$ and $N$ where $f(M) \subset[0, t]$ and $f(N) \subset[t, 1]\}$. Note that in particular $B(f)$ contains all of those points in $I$ whose point inverse has an empty or compact boundary. Finally let $F=\{f \in a: B(f)$ is dense in $I$ \}.

Lemma 1. Every $f \in F$ has a unique continuous extension $f$ to $\boldsymbol{\gamma} X$.

Proof. Let $f \in F$ and let $\left\{H_{1}, H_{2}, \cdots, H_{k}\right\}$ be a finite open cover-

Presented to the Society, January 24, 1967; received by the editors February 23, 1967. 
ing of $I$. Since $B(f)$ is dense in $I$ there exists a finite open cover $\left\{K_{1}, K_{2}, \cdots, K_{k}\right\}$ of $I$ such that $\bar{K}_{i} \subset H_{i}$ and $F r K_{i}$ is a finite subset of $B(f)$. It then follows from our definition of $B(f)$ that for each $i$ there exists an open set $G_{i}$ of $X$ such that $f^{-1}\left(K_{i}\right) \subset G_{i} \subset f^{-1}\left(H_{i}\right)$ and $F r G_{i}$ is compact. Thus if we consider $X$ as a uniform space with uniformity $\mathfrak{T}$, we have shown that $f$ is uniformly continuous. Hence $f$ is uniformly continuous and $f$ can be extended to a unique continuous mapping $f^{\prime}$ of $\gamma X$ into $I$.

The above proof is a modification of the proof of theorem 3 in [5] where K. Morita proves the following: Let $f$ be a closed mapping of a semicompact metric space $X$ onto a semicompact metric space $Y$. Then $f$ can be extended to a continuous mapping of $\gamma X$ onto $\gamma Y$.

REMARK. In [1, Theorem (3.1)] the author proved the following: Let $X$ be a locally connected generalized continuum. Then $X$ has the property that the complement of every compact set in $X$ has at most one nonconditionally compact component if and only if every $f \in F$ has a continuous extension to $X_{\infty}$, the one-point compactification of $X$. One can show that $\gamma X$ is topologically equivalent to $X_{\infty}$ if and only if $X$ has the property that the complement of every compact in $X$ has at most one nonconditionally compact component. Thus $\gamma X$ is topologically equivalent to $X_{\infty}$ if every $f \in F$ has a continuous extension to $X_{\infty}$.

Lemma 2. For every pair of disjoint closed subsets $A$ and $B$ of $\gamma X$ there exist an $f \in F$ and a continuous extension $f^{\prime}$ of $f$ to $\gamma X$ such that $f^{\prime}(A)=0$ and $f^{\prime}(B)=1$.

Proof. We will proceed to construct a mapping $f^{\prime}$ on $\gamma X$ separating $A$ and $B$ such that $f^{\prime} \mid X=f$ is in $F$. Let $D$ denote the set of positive dyadic rational numbers. Let $R_{1}=X-B$ and $R_{0}$ denote an open subset of $\gamma X$ containing $A$ such that the closure of $R_{0}$ misses $B$ and the boundary of $R_{0}$ lies entirely in $X$. We then proceed as in the proof of Urysohn's Lemma in [7, p. 115] and select for each $t \in D$ an open set $R_{t}$ of $\gamma X$ containing $A$ such that $R_{t}=X$ for $t>1$, and $\bar{R}_{s} \subset R_{t}$ whenever $s, t$ are in $D$ and $s<t$ but with the additional requirement that for each $t \in D, \operatorname{Fr} R_{t} \subset X$. Then if we define $f^{\prime}(x)=\inf \left\{t: x \in R_{t}\right\}$ we have that $f^{\prime}$ is a continuous function such that $f^{\prime}(A)=0, f^{\prime}(B)=1$ and for each $t \in D, F r R_{t} \subset F r f^{\prime-1}(t)$. Hence $f=f^{\prime} \mid X$ is in $F$ as required.

Theorem 1. The Freudenthal compactification $\gamma X$ of $X$ satisfies the following:

(i) Every $f \in F$ has a unique continuous extension $f^{\prime}$ to $\gamma X$; and

(ii) For every pair of distinct points $x$ and $y$ in the closure of $\gamma X-X$ there exists an $f \in F$ such that $f^{\prime}(x) \neq f^{\prime}(y)$. 
Furthermore $\gamma X$ is the topologically unique smallest Hausdorff compactification of $X$ satisfying (i) and $\gamma X$ is the topologically unique largest Hausdorff compactification of $X$ satisfying (ii). Hence $\gamma X$ is the topologically unique Hausdorff compactification of $X$ satisfying (i) and (ii).

Proof. By Lemmas 1 and 2, $\gamma X$ satisfies (i) and (ii). The minimality, maximality and uniqueness of $\gamma X$ follows immediately from the results in $\$ 5$ in [2].

REMARK. Let $P$ denote the product space $I^{F}$ and define an embedding $e$ of $X$ in to $P$ by $\left(\Pi_{f} \circ e\right)(x)=f(x)$ for $x \in X$ and $f \in F$. (Here $\Pi_{f}$ is the projection of $P$ onto the $f$ th coordinate.) Then if $\psi X$ denotes the closure of $e(X)$ in $P, \psi X$ is a Hausdorff compactification of $X$ satisfying (i) and (ii). Thus $\psi X$ and $\gamma X$ are topologically equivalent and we have shown that the Freudenthal compactification of a semicompact Hausdorff space can be obtained as a Tychonoff-type embedding.

When $X$ is a locally compact Hausdorff space one can replace $F$ by the set of all (closed) mappings of $X$ into $I$ that have compact boundaries of point inverses.

The author has shown that when $X$ is locally compact there exists a Hausdorff compactification $\alpha_{F} X$ of $X$ satisfying (i) and (ii) with respect to any arbitrary family of mappings of $X$ into any compact Hausdorff space $Y$. Thus it may be of interest to investigate the properties of compactifications one obtains when $F$ is replaced by certain other classes of mappings.

\section{REFERENCES}

1. R. F. Dickman, Jr., Unicoherence and related properties, Duke Math. J. 34 (1967), 343-352.

2. - Minimum and maximum compactifications of arbitrary topological spaces, Trans. Amer. Math. Soc. (to appear).

3. H. Freudenthal, Neuaufbau der Endentheorie, Ann. of Math. (2) 43 (1942), 261279.

4. K. Morita, On bicompactifications of semibicompact spaces, Sci. Rep. Tokyo Bunrika Daigaku Sect. A 4 (1952), 222-229.

5. - On images of an open interval under closed continuous mappings, Proc. Japan Acad. 32 (1959), 15-19.

6. J. R. Isbell, Uniform spaces, Math. Surveys, no. 12, Amer. Math. Soc., Providence, R. I., 1964.

7. J. L. Kelley, General topology', Van Nostrand, New York, 1955.

UNIVERSITy OF MiAMI 